\title{
REVISED Case Report: Rare presentation of De Garengeot Hernia
}

\section{[version 2; peer review: 1 approved, 1 approved with}

\section{reservations]}

\section{Tom Crawley-Smith (iD}

Rural Clinical School (Faculty of Medicine), University of Queensland, Herston, Brisbane, Queensland, 4029, Australia

V2 First published: 23 Jan 2019, 8:91

https://doi.org/10.12688/f1000research.16646.1

Latest published: $05 \mathrm{Jul}$ 2019, 8:91

https://doi.org/10.12688/f1000research.16646.2

\section{Abstract}

The presentation of an incarcerated appendix within a femoral hernia accounts for $0.5-3.3 \%$ of all femoral hernias. It is rarely apparent diagnostically prior to surgery. A 48-year-old female had a delayed presentation with a 3-day history of an irreducible right inguinal swelling. Imaging failed to elucidate an incarcerated appendix, which was found at operation. The patient made a full recovery. The rarity and presentation of this condition is discussed. On literature review it typically is not suspected at operation due to its rarity and the difficulty of interpreting it on examination and imaging.

Keywords

Femoral hernia, Incarceration, appendicitis, De Garengeot Hernia

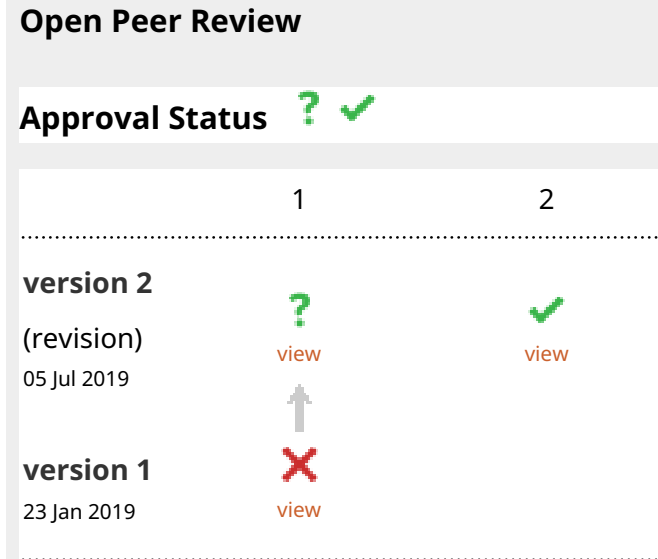

1. Zhamak Khurgami iD, The University of Oklahoma, Tulsa, USA

2. Samee Abdus (D), Royal Oldham Hospital, Pennine Acute Hospitals NHS Trust, Oldham, UK

Any reports and responses or comments on the article can be found at the end of the article.

Corresponding author: Tom Crawley-Smith (tomcrawley77@hotmail.com)

Author roles: Crawley-Smith T: Conceptualization, Writing - Original Draft Preparation, Writing - Review \& Editing

Competing interests: No competing interests were disclosed.

Grant information: The author(s) declared that no grants were involved in supporting this work.

Copyright: @ 2019 Crawley-Smith T. This is an open access article distributed under the terms of the Creative Commons Attribution License, which permits unrestricted use, distribution, and reproduction in any medium, provided the original work is properly cited.

How to cite this article: Crawley-Smith T. Case Report: Rare presentation of De Garengeot Hernia [version 2; peer review: 1 approved, 1 approved with reservations] F1000Research 2019, 8:91 https://doi.org/10.12688/f1000research.16646.2

First published: 23 Jan 2019, 8:91 https://doi.org/10.12688/f1000research.16646.1 


\section{REVISED Amendments from Version 1}

In this version 2, three figures have been added, we give further description of femoral hernia repair, and discuss imaging and operative management of the case.

See referee reports

\section{Introduction}

The presentation of femoral hernia with an incarcerated appendix accounts for $0.5-3.3 \%$ of all femoral hernias ${ }^{1}$; very few cases have been described ${ }^{2}$. The condition is named after Rene De Garengeot, a French surgeon who first described it in $1731^{3}$. The condition may be described as the femoral counterpart of the more widely described Amyand hernia, involving appendicitis within the inguinal hernia $\mathrm{sac}^{1}$.

\section{Patient information}

A 48-year-old woman presented to Dalby Hospital (a small rural facility) with a 3-day history of an irreducible right inguinal swelling, which came on while cycling a mountain bike. A timeline of care is given in Table 1. She had initially not presented as she suspected a muscle strain but presented when the pain became worse. She reported 2-3 previous occurrences of a lump in the same location many years ago, which had self-resolved. Her prior medical history was notable: 13 previous pregnancies with 10 natural deliveries and 3 terminations, LLETZ procedure for cervical cancer, no previous abdominal surgeries. She was an active smoker with a 25 pack-year history. She took no regular medications.

\section{Clinical examination}

The patient was initially examined by a rural general practitioner who was concerned for incarcerated hernia. He discussed the case with the surgical registrar at the treating regional hospital and arranged for interhospital transfer. On transfer

\section{Table 1. Timeline of patient events.}

\begin{tabular}{|l|l|l|}
\hline Day/week & Time & Event \\
\hline Day 0 & 1600 & Presented to Dalby Regional hospital \\
\hline & 2100 & Transferred to Toowoomba Hospital \\
\hline Day 1 & 0800 & Ultrasound scan ordered \\
\hline & 1200 & $\begin{array}{l}\text { CT Scan performed } \\
\text { Femoral hernia repair, appendicectomy } \\
\text { Placed on IV Ceftriaxone 1g D } \\
\text { IV Flagyl 50Omg BD } \\
\text { PO Analgesia }\end{array}$ \\
\hline Day 2 & & $\begin{array}{l}\text { Diet upgraded to Free fluids } \\
\text { Physiotherapy, mobilised }\end{array}$ \\
\hline Day 3 & Antibiotics ceased. Discharged \\
\hline Week 4 & $\begin{array}{l}\text { Pt reviewed in clinic. No recurrence, } \\
\text { wounds fully healed. }\end{array}$ \\
\hline
\end{tabular}

that evening to this regional facility the swelling was red and inflamed. The patient was haemodynamically stable, afebrile and was moving her bowels. A right sided, painful swelling could be palpated in the right inguinal region. The registrar examining the patient was suspicious for an incarcerated femoral hernia. In the absence of obstructive symptoms it was suspected that this was incarcerated fat only.

\section{Investigations}

In order to exclude the more serious diagnosis of incarcerated bowel with the hernia and to confirm the diagnosis of femoral hernia, an initial ultrasound was ordered by the treating surgical registrar after discussion with the consultant of the night. In addition a full blood count and electrolytes with liver function tests was ordered. The blood tests were all in the normal range. Meanwhile the ultrasound was unable to exclude incarcerated bowel and femoral from inguinal hernia (Figure 1). The discrepancy of this radiological finding with the clinical findings caused further discussion between the radiology sonographer, consultant and the surgical team; the diagnosis of the type of hernia and its contents mandated the urgency of theatre, approaches and timing. As a result, a CT was ordered to further investigate the anatomy in this case in order to plan operative approach.

The initial findings of the CT scan were suggestive of an inflamed inguinal hernia with predominant fat contents and probable bowel involvement (Figure 2 and Figure 3). There was no radiographic evidence of a small or large bowel obstruction. As this patient's bowels were still moving, it was felt that this was most likely caused by incarcerated, strangulated fat, rather than bowel.

The patient was taken to theatre. Because of the above findings, an incision was made over the inguinal ligament, expecting to find an incarcerated inguinal hernia. Instead, on dissection, a

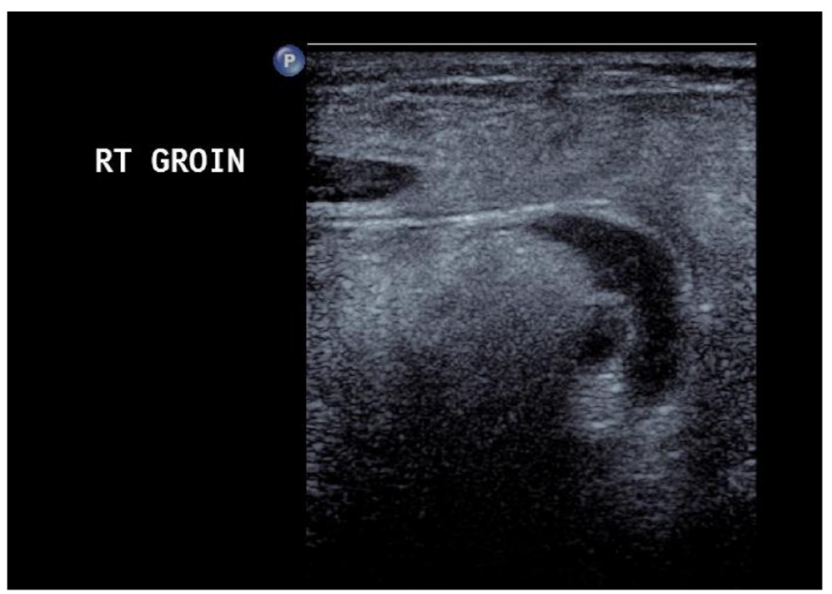

Figure 1. Ultrasound groin. The ultrasound failed to confidently describe the hernia as inguinal or femoral and was unable to exclude the presence of bowel involvement within the hernia. 


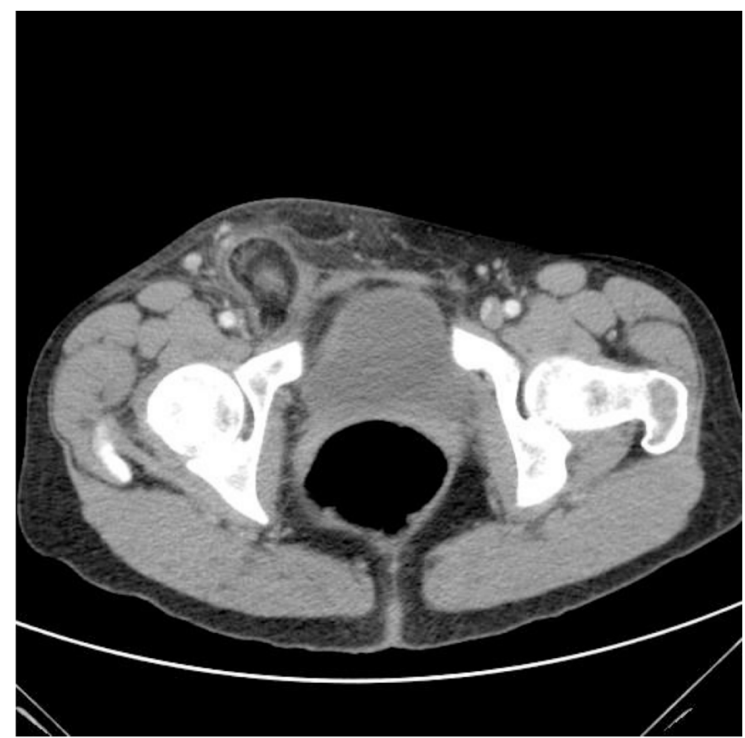

Figure 2. Hernial sac. This was reported at the time as an inguinal hernia with bowel involvement but without signs of small bowel obstruction. We were suspicious clinically of a femoral hernia and no bowel involvement.

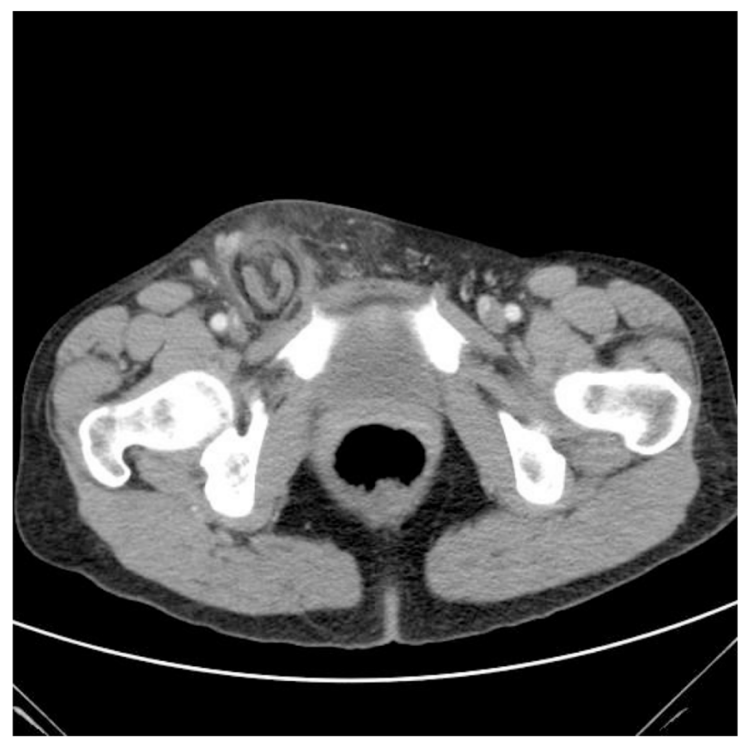

Figure 3. Contents of femoral sac: in retrospect this was the incarcerated appendix. As reported at the time it was reported as probable bowel involvement within an inguinal hernia.

femoral hernia was encountered. The sack was opened and necrotic mucosal content was encountered. It was suspected that this was necrotic bowel requiring resection, so the decision was made a low midline laparotomy to ensure safe resection. On opening, a necrotic appendix was found to be incarcerated in the femoral hernia (Figure 4).

The appendix being reduced, the mesoappendix was clamped, divided and ligated, and the appendix was removed with a purse

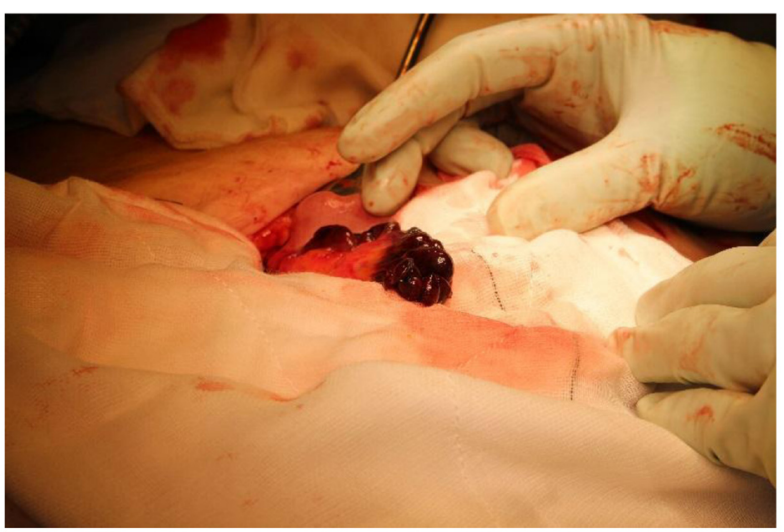

Figure 4. Findings at laparotomy; inflamed infarcted appendix.

string suture used to bury residual mucosa. The femoral hernia was repaired primarily with nylon sutures from to the conjoin tendon to the shelf of the inguinal ligament after excision of the sac. A Blake drain was placed and laparotomy wounds were closed with looped Novafil sutures. The patient was placed on Ceftriaxone, $1 \mathrm{~g}$ daily and metronidazole $500 \mathrm{mg}$ BD.

The patient recovered swiftly, and was discharged on day 3 following surgery (Table 1). Her antibiotics were ceased after 24 hours and she was treated with simple analgesia only as required. She was subsequently seen in the outpatients' clinic at 2 weeks after surgery, and had made a full recovery.

\section{Discussion}

This case was notable for its rarity and the clinical and radiological difficulty anticipating the incarceration of the appendix in the femoral canal. This might have mandated a different approach on surgery than might have been undertaken. Accurate diagnosis of the condition would allow for appropriate choosing of incision, or a laparoscopic approach. On review of the literature, this is typical of this rare condition, however. Excepting one Japanese study $^{4}$, the diagnosis was typically made serendipitously at surgery. This is not unique, to De Garengeot's hernia; there can often be confusion between femoral hernia and inguinal hernias, particularly upon clinical examination. Littre's hernia containing Meckel's diverticulum and a Richter's hernia are often also diagnosed on the table rather than in the radiologist's suite.

The rarity of this condition contributes to this. De Garengeot's is an exceedingly rare condition, accounting for $0.5-3.3 \%$ of femoral hernias ${ }^{1}$, which are rare in and of themselves, accounting for only $3-5 \%$ of all presentations of hernia. This can make clinical and radiological suspicion all the more challenging. In this case despite ultrasound and CT scan the operative approach was as for an inguinal hernia without incarcerated bowel. We anticipated using mesh in this case but elected not to in the presence of an infarcted inflamed appendix.

The suspicion of a De Garengeot's hernia earlier on imaging would have dictated either a laparoscopic approach, which may 
have also been useful to confirm the diagnosis, or a midline mini-laparotomy. Laparoscopic repair has been described as feasible for treatment of this condition; however, its rarity and difficulty of diagnosis prior to operation would make prospective comparison extremely difficult.

\section{Data availability}

No data are associated with this article.

\section{Consent}

Written consent for publication of their clinical details and clinical images was obtained from the patient.

Grant information

The author(s) declared that no grants were involved in supporting this work.

References

1. Voitk AC, McFarlane JK, Estrada RL: Ruptured appendicitis in femoral hernias: report of two cases and review of the literature. Ann Surg. 1974; 179(1): 24-26.

PubMed Abstract | Publisher Full Text | Free Full Text

2. Talini C, Oliveira LO, Araújo AC, et al.: De Garengeot hernia: Case report and review. Int J Surg Case Rep. 2015; 8C: 35-37.

PubMed Abstract | Publisher Full Text | Free Full Text
3. Gillion JF, Bornet G, Hamrouni A, et al.: Amyand and de Garengeot' hernias. Hernia. 2007; 11(3): 289-290.

PubMed Abstract | Publisher Full Text

4. Ebisawa K, Yamazaki S, Kimura Y, et al:: Acute Appendicitis in an Incarcerated Femoral Hernia: A Case of De Garengeot Hernia. Case Rep Gastroenterol. 2009;

3(3): 313-317.
PubMed Abstract | Publisher Full Text | Free Full Text 


\section{Open Peer Review}

\section{Current Peer Review Status:}

\section{Version 2}

Reviewer Report 08 October 2019

https://doi.org/10.5256/f1000research.20517.r50856

(C) 2019 Abdus S. This is an open access peer review report distributed under the terms of the Creative Commons Attribution License, which permits unrestricted use, distribution, and reproduction in any medium, provided the original work is properly cited.

\section{Samee Abdus}

Department of Surgery, Royal Oldham Hospital, Pennine Acute Hospitals NHS Trust, Oldham, UK

A good case report highlighting a rare entity and clinician should be aware of such condition.

Overall well written.

Concise.

Role of pre-op MRI for diagnosis? You may add up a line citing the ref Halpenny et al. (2012) ${ }^{1}$

Please correct the spelling "The sack was opened and necrotic mucosal..." it should be "sac".

Can you remove this bit? "As patients's bowel were still moving". Bowel movement or nonmovement has no value while making a clinical decision and is irrelevant. It is not uncommon for patients to break wind or open bowel even in cases of complete obstruction

Can be indexed with minor amendments.

\section{References}

1. Halpenny D, Barrett R, O'Callaghan K, Eltayeb O, et al.: The MRI findings of a de Garengeot hernia.BrJ Radiol. 2012; 85 (1011): e59-61 PubMed Abstract | Publisher Full Text

Is the background of the case's history and progression described in sufficient detail? Yes

Are enough details provided of any physical examination and diagnostic tests, treatment given and outcomes?

Yes 
Is sufficient discussion included of the importance of the findings and their relevance to future understanding of disease processes, diagnosis or treatment?

Yes

Is the case presented with sufficient detail to be useful for other practitioners?

Yes

Competing Interests: No competing interests were disclosed.

Reviewer Expertise: Molecular genetics, academic and clinical surgery.

I confirm that I have read this submission and believe that I have an appropriate level of expertise to confirm that it is of an acceptable scientific standard.

Reviewer Report 12 July 2019

https://doi.org/10.5256/f1000research.20517.r50814

(c) 2019 Khurgami Z. This is an open access peer review report distributed under the terms of the Creative Commons Attribution License, which permits unrestricted use, distribution, and reproduction in any medium, provided the original work is properly cited.

\section{Zhamak Khurgami}

Department of Surgery, College of Medicine, The University of Oklahoma, Tulsa, OK, USA

Revisions were noted. Regarding the surgical technique, the authors mentioned "Suturing the conjoin tendon to the shelf of the inguinal ligament after excision of the sac" which is a Bassini repair. This repair may not address the femoral hernia.

Is the background of the case's history and progression described in sufficient detail? Yes

Are enough details provided of any physical examination and diagnostic tests, treatment given and outcomes?

Yes

Is sufficient discussion included of the importance of the findings and their relevance to future understanding of disease processes, diagnosis or treatment?

Yes

Is the case presented with sufficient detail to be useful for other practitioners?

Yes

Competing Interests: No competing interests were disclosed. 
Reviewer Expertise: Surgery, gastrointestinal diseases.

I confirm that I have read this submission and believe that I have an appropriate level of expertise to confirm that it is of an acceptable scientific standard, however I have significant reservations, as outlined above.

\section{Version 1}

Reviewer Report 08 March 2019

https://doi.org/10.5256/f1000research.18192.r45339

(C) 2019 Khurgami Z. This is an open access peer review report distributed under the terms of the Creative Commons Attribution License, which permits unrestricted use, distribution, and reproduction in any medium, provided the original work is properly cited.

\section{Zhamak Khurgami}

Department of Surgery, College of Medicine, The University of Oklahoma, Tulsa, OK, USA

The table could be deleted, and the table information explained in the text.

The names of all the hospitals are not necessary to mention.

Please provide a picture from the CT scan if possible.

Please discuss if the patient could be managed without the use of US and CT scans and just explore the incarcerated hernia.

Please discuss if, based on available evidence, we could use the mesh in this situation.

Please explain which structure was sutured to the conjoint tendon.

Many details in the presentation and technique could be deleted.

Is the background of the case's history and progression described in sufficient detail? Yes

Are enough details provided of any physical examination and diagnostic tests, treatment given and outcomes?

Partly

Is sufficient discussion included of the importance of the findings and their relevance to future understanding of disease processes, diagnosis or treatment?

Partly

Is the case presented with sufficient detail to be useful for other practitioners?

Yes

Competing Interests: No competing interests were disclosed. 
Reviewer Expertise: Surgery, gastrointestinal diseases

I confirm that I have read this submission and believe that I have an appropriate level of expertise to state that I do not consider it to be of an acceptable scientific standard, for reasons outlined above.

Author Response 25 Mar 2019

Tom Crawley-Smith, University of Queensland, Herston, Brisbane, Australia

Thank you for your feedback.

I agree with the comment regarding the table. I originally did supply the time course within the text but was told to include this as a timeline by the journal for submission. I have included it but would be very happy to revert to how it was previously.

I have added images from the CT, Ultrasound and intra operative findings which hopefully illuminate the case. Ultimately we found the imaging did not actually impact our decision to take the patient to theatre or help us with deciding on initial incision. In retrospect it could be argued simply proceeding to theatre without imaging would have been an equal and superior outcome in terms of time. I have adjusted my comments to this in the discussion.

In terms of our femoral hernia repair after the excision of the incarcerated appendix, we performed a Bassini repair by suturing the conjoin tendon to the inguinal ligament to close the potential space with nylon. Mesh repair was considered but given the resection of the appendix it was felt to be too high a potential risk of infection

Competing Interests: No competing interests were disclosed.

The benefits of publishing with F1000Research:

- Your article is published within days, with no editorial bias

- You can publish traditional articles, null/negative results, case reports, data notes and more

- The peer review process is transparent and collaborative

- Your article is indexed in PubMed after passing peer review

- Dedicated customer support at every stage

For pre-submission enquiries, contact research@f1000.com

F1000Research 